\title{
CONTROLE QUÍMICO DE BONAGOTA SALUBRICOLA (MEYRICK, 1937) (LEPIDOPTERA: TORTRICIDAE) EM LABORATÓRIO E POMAR DE MACIEIRA
}

\author{
M. Botton ${ }^{1}$, C.J. Arioli ${ }^{2}$, R. Ringenberg ${ }^{3}$, W.J. Morandi Filho ${ }^{1}$ \\ ${ }^{1}$ Embrapa Uva e Vinho, Rua Livramento, 515, CEP 95700-000, Bento Gonçalves, RS, Brasil. E-mail: marcos@ \\ cnpuv.embrapa.br
}

RESUMO

\begin{abstract}
A lagarta-enroladeira Bonagota salubricola é considerada uma das principais pragas da macieira no Brasil. Para o controle do inseto, tem sido empregado o inseticida clorpirifós-etil, porém, o produto encontra restrições de uso, principalmente devido à toxicidade e a efeitos secundários sobre inimigos naturais. Com o objetivo de avaliar novos inseticidas que possam ser empregados no controle da lagarta-enroladeira, foram conduzidos experimentos de laboratório e em pomar comercial. Os inseticidas avaliados foram spinosad (Tracer 480 SC, 20 e $25 \mathrm{~mL} / 100 \mathrm{~L}$ ), metoxifenozide (Intrepid 240 SC, 40 e 60 mL/100 L), benzoato de emamectina (Proclaim 5 SG, 10 e 15 g/ 100 L) associado ao espalhante adesivo AgBem $250 \mathrm{~mL} / 100 \mathrm{~L}$ e etofenproxi (Trebon $100 \mathrm{SC}$, 100 e 150 mL/100 L). Para comparação, foi utilizado o clorpirifós-etil (Lorsban 480 CE, 150 mL/100 L) e uma testemunha. Em laboratório, os inseticidas foram aplicados sobre posturas do inseto (com até 48 horas) e em folhas de maçãs, com posterior infestação de lagartas. Nenhum inseticida apresentou efeito sobre posturas da espécie. Os inseticidas benzoato de emamectina $(0,5$ e 0,75 g/100 L), clorpirifós-etil (75 g/100 L), metoxifenozide (14,4 g/100 L) e spinosad (12 g/100 L) provocaram mortalidade de lagartas superior a 90\%, 96 horas após a aplicação. O etofenproxi não apresentou mortalidade significativa. Em pomar comercial, o dano nos frutos foi significativamente reduzido por todos os inseticidas, sendo que os produtos benzoato de emamectina $(0,5$ e 0,75 g/100 L) e metoxifenozide $(14,4 \mathrm{~g} / 100 \mathrm{~L})$ apresentaram resultado equivalente ao inseticida clorpirifós-etil $(75 \mathrm{~g} / 100 \mathrm{~L})$, reduzindo a porcentagem de frutos danificados em $85 \%$. O inseticida spinosad (12 $\mathrm{g} / 100 \mathrm{~L}$ ) e o etofenproxi ( $10 \mathrm{e} 15 \mathrm{~g} / 100 \mathrm{~L}$ ) reduziram os danos em 75,6 e $45 \%$, respectivamente. Os inseticidas benzoato de emamectina e metoxifenozide são alternativas ao uso do clorpirifós-etil no controle da lagarta-enroladeira na cultura da macieira.
\end{abstract}

PALAVRAS-CHAVE: Lagarta-enroladeira da maçã, injúria, Malus sp.

\begin{abstract}
CHEMICAL CONTROL OF BONAGOTA SALUBRICOLA (MEYRICK, 1937) (LEPIDOPTERA: TORTRICIDAE) IN THE LABORATORY AND APPLE ORCHARD. The Brazilian apple leafroller (BAL) Bonagota salubricola is one of the major apple pests in Brazil. For pest control, chlorpyriphosethyl is the principal compound used by growers, however its use entails environmental and health concerns. The objective of this work was to evaluate new insecticides for BAL control in laboratory and field experiments. Insecticides evaluated were spinosad (Tracer $480 \mathrm{SC}, 20$ and $25 \mathrm{~mL} / 100 \mathrm{~L}$ ), methoxyfenozide (Intrepid 240 SC, 40 and $60 \mathrm{~mL} / 100 \mathrm{~L}$ ), emamectin benzoato (Proclaim 5 SG, 10 and $15 \mathrm{~g} / 100 \mathrm{~L}$ ) associated with the adjuvant AgBem, $250 \mathrm{~mL} / 100 \mathrm{~L}$, and etofenproxi (Trebon 100 SC, 100 and $150 \mathrm{~m} \mathrm{~L} / 100 \mathrm{~L}$ ). Chlorpyriphos-ethyl (Lorsban $480 \mathrm{CE}, 150 \mathrm{~mL} / 100 \mathrm{~L}$ ) was used as a standard. In the laboratory, the insecticides were applied on egg masses (48 hours old) and apple leaves with posterior larvae infestation. No tested insecticide provided ovicidal effect. The insecticides emamectin benzoato $(0.5$ and $0.75 \mathrm{~g} / 100 \mathrm{~L})$, chlorpyriphos-ethyl $(75 \mathrm{~g} / 100 \mathrm{~L})$, methoxyfenozide (14,4 g/100 L) and spinosad (12 g/100 L) caused larvae mortality above 90\%, 96 hours after the application. Etofenproxi had reduced effects on larvae. In a commercial orchard, the damage in fruits was reduced for all treatments. The insecticides emamectin benzoato $(0.5$ and $0.75 \mathrm{~g} / 100 \mathrm{~L}$ ) and methoxyfenozide $(14.4 \mathrm{~g} / 100 \mathrm{~L}$ ) were equivalent to chlorpyriphos-ethyl (75 $\mathrm{g} / 100 \mathrm{~L})$, reducing the damage by $85 \%$. The insecticide spinosad $(12 \mathrm{~g} / 100 \mathrm{~L})$ and etofenproxi (10
\end{abstract}

${ }^{2}$ Isca Tecnologias, Vacaria, RS, Brasil.

${ }^{3}$ Escola Superior de Agricultura "Luiz de Queiroz", Piracicaba, SP, Brasil. 
and $15 \mathrm{~g} / 100 \mathrm{~L}$ ) provided 75.6 and $45 \%$ reduction of fruit damage, respectively. The insecticides emamectin benzoato and methoxyfenozide are alternatives to chlorpyriphos-ethyl use for the control of BAL in apple orchards.

KEY WORDS: Brazilian apple leafroller, Malus sp., injuries.

\section{INTRODUÇÃO}

A lagarta-enroladeira Bonagota salubricola (Lepidoptera:Tortricidae), anteriormente conhecida como Bonagota cranaodes (BRown; RAzOWsKI, 2003), é uma das mais recentes pragas da macieira no Brasil (Kovaleski et al., 1998). As primeiras infestações foram relatadas na década de 80 (LoRENZATO, 1984), sendo que, na safra de 1984, verificou-se o primeiro grande ataque, principalmente sobre a cultivar Fuji, cuja produção é mais tardia (KovALESKI, 1994).

A espécie é considerada polífaga, atacando um grande número de plantas cultivadas e silvestres. Além da macieira, o inseto foi observado alimentando-se de álamo, ameixeira, hortênsia, nabo, pereira, roseira, serralha e trevo (KOVALESKI et al., 1998). Os adultos são de coloração cinza-claro, medindo cerca de $15 \mathrm{~mm}$ de envergadura e de 7 a $10 \mathrm{~mm}$ de comprimento. As fêmeas colocam seus ovos na superfície adaxial da folha de macieira e de outras espécies hospedeiras. eles são depositados em grupos contendo em média 40 ovos, num total de 200 por fêmea, sendo que a duração do período de ovo a adulto é, em média, 42 dias a $25^{\circ} \mathrm{C}$ (KovaleskI et al., 2003).

$\mathrm{O}$ ataque do inseto pode ser visualizado nas folhas e frutos, sendo que nas folhas não há perdas econômicas (KOVALESKI et al., 1998). Os principais prejuízos ocorrem quando as lagartas raspam a casca dos frutos, depreciando-os comercialmente. Devido ao seu ataque, pode haver perdas de até $9,6 \%$ na produção (KOVALESKI I, 1994). Nos últimos anos, com a utilização de feromônios sexuais sintéticos para o monitoramento da praga nos pomares e a aplicação de inseticidas fosforados para o controle do inseto, as perdas estão entre 3 a $5 \%$, podendo ser reduzidas a $1 \%$, principalmente, na região de Vacaria, maior pólo produtor de maçãs do Estado do Rio Grande do Sul (Kovaleski; Ribeiro, 2003).

Embora técnicas como a confusão sexual estejam sendo avaliadas visando o controle do inseto nos pomares comerciais (PASTORI, 2007), os produtores têm empregado basicamente o controle químico, sendo o inseticida clorpirifós-etil o composto mais eficiente (BotTon et al., 2000; Kovaleski; Ribeiro, 2003). Entretanto, existe uma preocupação crescente entre técnicos e produtores para racionalizar e/ ou substituir o emprego de inseticidas fosforados, especialmente nos agroecossistemas frutícolas conduzidos sob sistemas de produção integrada (Tітा et al.,1995). Entre os motivos que exigem a necessidade urgente de substituir este grupo químico na fruticultura, destacam-se os efeitos secundários sobre a entomofauna benéfica, riscos de intoxicação dos aplicadores e contaminação ambiental. Além disso, são cada vez maiores as restrições quanto ao uso de inseticidas fosforados devido à carência elevada, principalmente quando empregados no período de pré-colheita.

Tendo em vista a baixa disponibilidade de grupos químicos para uso no manejo de pragas na cultura da macieira, torna-se necessário avaliar novos inseticidas mais eficientes para o controle da lagarta-enroladeira, bem como definir estratégias para o seu emprego em pomares comerciais. Dentre as novas alternativas para o controle químico do inseto, surgem os lagarticidas específicos, como o metoxifenozide e o benzoato de emamectina (JANSSON et al., 1997; Sun et al., 2000). Como vantagens destes produtos, citam-se a dosagem reduzida, baixa toxicidade e carência, além da especificidade sobre lagartas, evitando efeitos tóxicos sobre organismos não-alvo (Morando et al., 1990; Leibee et al., 1995; CARLson et al., 2001). Além dos inseticidas específicos para o controle de lepidópteros, é fundamental que sejam avaliadas alternativas para substituir o carbaril e o dimetoato, produtos amplamente empregados no período de pré-colheita das maçãs, quando ocorre ataque conjunto da mosca-das-frutas Anastrepha fraterculus (Diptera: Tephritidae). Na linha dos novos inseticidas substitutos dos fosforados que possuem potencial para serem empregados nessa fase, destacam-se o spinosad, produto derivado da fermentação do actinomiceto Sacharopolyspora spinosa (SPARKs et al., 1998; Thompson; Hutchins, 1999) e o etofenproxi, composto à base de carbono, oxigênio e hidrogênio (Yоsнімото et al., 1989).

Este trabalho foi realizado com o objetivo de avaliarem-se novos inseticidas que possam ser utilizados como substitutos dos fosforados no controle da lagarta-enroladeira $B$. salubricola na cultura da macieira.

\section{MATERIAL E MÉTODOS}

A avaliação de inseticidas para o controle de $B$. salubricola foi conduzida no Laboratório de Entomologia da Embrapa Uva e Vinho, localizado em Bento 
Gonçalves, RS (latitude $29^{\circ} 09^{\prime} 44^{\prime \prime}$ Sul, longitude $51^{\circ} 31^{\prime} 50^{\prime \prime}$ Oeste), e em pomar comercial de macieira da cv. Fuji, localizado no Município de Vacaria, RS (latitude $28^{\circ} 30^{\prime}$ Sul, longitude $50^{\circ} 54^{\prime}$ Oeste), no perío-do dejaneiro a abril de 2003, quando as plantas estavam em período de produção.

Os seguintes tratamentos foram avaliados tanto nolaboratório como no pomar comercial: etofenproxi (Trebon 100 SC, 100 e 150 mL/100 L), benzoato de emamectina (Proclaim 5 SG, 10 e 15 g/100 L) associado a espalhante adesivo (AgBen, $250 \mathrm{~mL} / 100$ L), clorpirifós-etil (Lorsban 480 CE, 150 mL/100 L), metoxifenozide (Intrepid 240 SC, 40 e $60 \mathrm{~mL} / 100$ L) e spinosad (Tracer $480 \mathrm{SC}, 20$ e $25 \mathrm{~mL} / 100 \mathrm{~L}$ ). O tratamento testemunha foi constituído somente por água.

Experimento de laboratório. O experimento de laboratório foi conduzido na temperatura de $25 \pm$ $3^{\circ}$ C, U.R. de $80 \pm 10 \%$, utilizando-se insetos criados em dieta artificial seguindo a metodologia descrita por PARRA et al. (1995). O efeito dos produtos sobre ovos foi verificado utilizando-se posturas com até 48 horas de idade, as quais foram mergulhadas nas respectivas caldas inseticidas. Cada tratamento constou de dez repetições (uma postura por repetição), tendo sido os ovos contados antes do tratamento. A avaliação da eclosão das lagartas foi realizada 12 dias após o tratamento.

Oefeito sobre lagarticida foi realizado utilizando-selagartas $(\geq 1,5 \mathrm{~cm})$ de $B$. salubricola, sendo quecada parcela experimental foi composta por um pote de plástico transparente com tampa $(5,0 \times 10,0 \mathrm{~cm}$ de altura e diâmetro, respectivamente), estabelecendo-secinco repetições no delineamento experimental inteiramente casualizado. Discos de folhas de macieira ( $3,5 \mathrm{~cm}$ de diâmetro) da cv. Fuji foram obtidos com o auxílio de um vazador e, em seguida, pulverizados na face abaxial e adaxial em torre de pulverização (Burkard Scientific Uxbridge UK). A pressão de trabalho foi de $10 \mathrm{lb} / \mathrm{pol}^{2}$, utilizando-se $1 \mathrm{~mL}$ de calda por pulverização, obtendo-se uma deposição média deresíduo úmido de $1,8 \mathrm{mg} / \mathrm{cm}^{2}$. Após o tratamento, os discos foram deixados por duas horas, à sombra, para secagem da calda. Em cada pote de plástico (repetição) foram colocados quatro discos e quatro lagartas. Os discos foram sobrepostos para reduzir a perda de umidade e proporcionar abrigo para as lagartas. A mortalidade foi avaliada 24, 48, 72 e 96 horas após a infestação.

Experimento em pomar comercial.Oexperimento para avaliar o efeito de inseticidas foi conduzido em Vacaria, RS, em pomar comercial de macieiras da cv. Fuji, com oito anos de idade, enxertadas sobre M-9 e plantadas no espaçamento de 3,5 x 1,5 m e com altura aproximada de 2,5 a 3,0 m. A adubação, controle de doenças e manejo de plantas invasoras foram realizados conforme as recomendações con- tidas nas normas para a produção integrada para a cultura da macieira (PROTAS; SANHUEZA, 2000). Os inseticidas foram aplicados com o auxílio de um turbo atomizador, utilizando um volume de $900 \mathrm{~L}$ de calda por hectare, sendo que cada tratamento foi aplicado numa área aproximada de $0,3 \mathrm{ha}$. As aplicações foram realizadas no período de maturação dos frutos, nos dias 14 e 26/2, 13/3 e 25/3 e 8/4/2003, estabelecendo-se um intervalo entre os tratamentos de, aproximadamente, 12 dias. A avaliação do dano causado pela lagarta-enroladeira nos frutos foi realizada em 17/4/2003, colhendo-se 500 frutos em cinco pontos de amostragem (repetições) de 100 frutos cada no interior da área tratada.

Para a análise estatística, o número de lagartas vivas foi transformado em $(X+0,5)^{1 / 2}$ e a porcentagem de frutos danificados em arc seno $(\% / 100)^{1 / 2}$, objetivando normalizar a variância. As médias foram comparadas pelo teste de Tukey $(p<0,05)$ utilizando o programa Genes (CRUZ, 2001), sendo a porcentagem de mortalidade ou a redução de danos nos frutos calculada através da fórmula de Аввотт (1925).

\section{RESULTADOS E DISCUSSÃO}

Não foi constatado efeito ovicida quando os inseticidas foram aplicados sobre posturas de até 48 horas, sendo que a eclosão de lagartas nos tratamentos foi equivalente à testemunha (Tabela 1). Na avaliação realizada 24 horas após o tratamento, verificou-se que somente o clorpirifós-etil $(72 \mathrm{~g} / 100$ L) provocou $100 \%$ de mortalidade das lagartas de B. salubricola (Tabela 2). Os inseticidas benzoato de emamectina ( 0,5 e 0,75 g/100 L) e spinosad (9,6 e $12 \mathrm{~g} / 100 \mathrm{~L})$ provocaram mortalidade intermediária (45 a $65 \%)$, enquanto que o uso do etofenproxi (10 e $15 \mathrm{~g} / 100 \mathrm{~L})$ e do metoxifenozide $(9,6$ e 14,4 g/ 100 L) não resultou em diferenças significativas em relação ao tratamento testemunha (Tabela 2). $\mathrm{Na}$ segunda avaliação, realizada $48 \mathrm{HAT}$, constatou-se que os inseticidas benzoato de emamectina $(0,5 \mathrm{e}$ $0,75 \mathrm{~g} / 100 \mathrm{~L})$ e spinosad ( $12 \mathrm{~g} / 100 \mathrm{~L})$ causaram mortalidade equivalente ao clorpirifós-etil (72 $\mathrm{mL} / 100 \mathrm{~L}$ ), mantendo o mesmo comportamento na avaliação realizada 72 e 96 HAT (Tabela 2). O metoxifenozide $(14,4 \mathrm{~g} / 100 \mathrm{~L})$ somente foi equivalente ao inseticida clorpirifós-etil (75 g/100 L) na avaliação final, realizada $96 \mathrm{HAT}$, enquanto que a menor dosagem do spinosad (9,6 g/100 L) e do metoxifenozide $(9,6 \mathrm{~g} / 100 \mathrm{~L})$ possibilitaram controle próximo a $70 \%$ (Tabela 2 ). O inseticida etofenproxi (10 e $15 \mathrm{~g} / 100 \mathrm{~L})$, em todas as avaliações, não provocou mortalidade significativa de lagartas grandes de B. salubricola em laboratório (Tabela 2). 
Tabela 1 - Viabilidade de ovos de B. salubricola após a aplicação de inseticidas em posturas com até 48 horas de idade. Bento Gonçalves, RS, 2003.

\begin{tabular}{lcccc}
\hline Produto comercial (Ingrediente ativo) & \multicolumn{2}{c}{ Dose (g ou mL/100L) } & & \\
& i.a & p.c. & \% Eclosão & \% Redução ${ }^{2}$ \\
\hline Trebon 100 SC (Etofenproxi) & 10 & 100 & $81,7 \pm 8,84 \mathrm{a}$ & 5,4 \\
Trebon 100 SC (Etofenproxi) & 15 & 150 & $59,2 \pm 15,23 \mathrm{a}$ & 31,51 \\
Tracer 480 SC (Spinosad) & 9,6 & 20 & $79,6 \pm 7,85 \mathrm{a}$ & 7,8 \\
Tracer 480 SC (Spinosad) & 12,0 & 25 & $79,2 \pm 7,56 \mathrm{a}$ & 8,3 \\
Intrepid 240 SC (Metoxifenozide) & 9,6 & 40 & $69,8 \pm 10,33 \mathrm{a}$ & 19,2 \\
Intrepid 240 SC (Metoxifenozide) & 14,4 & 60 & $85,6 \pm 9,18 \mathrm{a}$ & 0,9 \\
Proclaim 5 SG (emamectina) & 0,5 & 10 & $57,6 \pm 13,33 \mathrm{a}$ & 33,4 \\
Proclaim 5 SG (emamectina) & 0,75 & 15 & $78,7 \pm 9,62 \mathrm{a}$ & 8,9 \\
Lorsban 480 BR (Clorpirifós-etil) & 72 & 150 & $3,2 \pm 1,50 \mathrm{~b}$ & 96,30 \\
Testemunha & - & - & $86,4 \pm 5,40 \mathrm{a}$ & -
\end{tabular}

${ }^{1}$ Médias não seguidas pela mesma letra diferem entre si pelo teste de Tukey, em nível de 5\% de probabilidade de erro. ${ }^{2}$ Porcentagem de redução na eclosão em comparação com a testemunha.

A diferença marcante na mortalidade das lagartas tratadas com metoxifenozide observada entre 24 e 96 HAT deve-se ao modo de ação do inseticida, que é mais lento que os produtos neurotóxicos (Hadialla et al., 1998). Os resultados obtidos com o clorpirifós-etil foram similares aos observados por BotTon et al. (2000), assim como o efeito do metoxifenozide apresentou resultado similar ao tebufenozide, produto atualmente empregado no controle de lagartas na cultura da macieira (KovALESKI; RIBEIRO, 2002).

Com base nestes resultados, verifica-se que os inseticidas spinosad (Tracer 480 SC, $25 \mathrm{~mL} / 100$ L), metoxifenozide (Intrepid 240 SC, $60 \mathrm{~mL} / 100$ L), benzoato de emamectina (Proclaim 5 SG, 10 e $15 \mathrm{~g} / 100 \mathrm{~L}$ ) associado ao espalhante adesivo AgBem 250 mL/100 L e clorpirifós-etil (Lorsban $480 \mathrm{CE}, 150 \mathrm{~mL} / 100 \mathrm{~L}$ ) apresentam atividade biológica sobre lagartas grandes de $B$. salubricola em laboratório.

Quando estes inseticidas foram empregados em pomar comercial, o dano provocado pela lagarta-enroladeira nas plantas testemunhas foi superior ao das protegidas pelos inseticidas, observando-se diferença significativa entre os produtos aplicados (Tabela 3). O menor dano foi constatado nos tratamentos com benzoato de emamectina $(0,5$ e 0,75 g/100 L), clorpirifós-etil (72 mL/100 L) e metoxifenozide $(9,6$ e 14,4 mL/100 L), os quais reduziram a porcentagem de frutos danificados em relação à testemunha, entre $85,4 \%$ e $97,6 \%$ conforme o produto e dose utilizada (Tabela 2). O spinosad (12 mL/100 L) reduziu o dano nos frutos em $75,6 \%$, enquanto que o etofemproxi $(10 \mathrm{e} 15 \mathrm{~g} / 100 \mathrm{~L})$ e o spinosad $(9,6$ $\mathrm{g} / 100 \mathrm{~L}$ ) proporcionaram uma redução próxima a $50 \%$ (Tabela 3).
Os resultados obtidos com o metoxifenozide e o benzoato de emamectina neste trabalho assemelham-se aos obtidos por ARIOLI et al. (2004) no controle de Grapholita molesta (Busck) na cultura do pessegueiro, em cujo experimento foram observadas porcentagens de controle em torno de $90 \%$ para ambos os inseticidas. Estes resultados são bastante interessantes dentro de um programa de manejo integrado de pragas, pois, na cultura da macieira, B. salubricola ocorre conjuntamente com G. molesta, sendo que ambas as espécies podem ser controladas com a utilização do metoxifenozide e o benzoato de emamectina, diminuindo, assim, o número de aplicações e a quantidade de ingrediente ativo utilizado por hectare.

Com relação aos inseticidas spinosad e etofenproxi, apesar de possuírem uma eficiência intermediária no controle de $B$. salubricola, contemplam um maior espectro de ação e poderiam ser direcionados para a fase de pré-colheita, controlando G. molesta e adultos de mosca-das-frutas sul-americana $A$. fraterculus (Wiedemann), que também ocorrem nesta fase (Scoz et al., 2004; ARIoli et al., 2004).

Com base nos resultados de laboratório e de pomar comercial, verificou-se que os inseticidas metoxifenozide (Intrepid 240 SC, 40 e $60 \mathrm{~mL} / 100$ L), benzoato de emamectina (Proclaim 5 SG, 10 e 15 $\mathrm{g} / 100 \mathrm{~L}$ ) associado ao espalhante adesivo AgBem $250 \mathrm{~mL} / 100 \mathrm{~L}$ e clorpirifós-etil (Lorsban $480 \mathrm{CE}$, $150 \mathrm{~mL} / 100 \mathrm{~L}$ ) reduzem com eficiência o dano da lagarta-enroladeira em pomares de macieira. Os inseticidas metoxifenozide e o benzoato de emamectina apresentam-se como novas alternativas para o controle da lagarta-enroladeira na cultura da macieira, apresentando menor toxicidade e reduzida dose de aplicação, quando comparados com os fosforados. 





Tabela 3 - Porcentagem de frutos danificados (\%FD) por lagartas de Bonagota salubricola e redução do dano em relação à testemunha (\%RFD) após cinco aplicações de inseticidas (14 e 26/2, 13 e 25/3 e 8/4/2003), em pomar comercial de macieiras da cultivar Fuji. Vacaria, RS, 2003.

\begin{tabular}{|c|c|c|c|c|c|}
\hline \multirow[t]{2}{*}{ Produto comercial } & \multirow[t]{2}{*}{ Ingrediente ativo } & \multicolumn{2}{|c|}{ Dose (g ou mL/100L) } & \multicolumn{2}{|c|}{ Avaliação $(17 / 4 / 2003)^{1}$} \\
\hline & & i.a & p.c. & $\% \mathrm{FD}^{2}$ & $\%$ (RFD) \\
\hline Trebon 100 SC & Etofenproxi & 10,0 & 100 & $4,4 \pm 1,21 \mathrm{abc}$ & 46,3 \\
\hline Trebon 100 SC & Etofenproxi & 15,0 & 150 & $4,8 \pm 1,20 \mathrm{ab}$ & 41,5 \\
\hline Tracer 480 SC & Spinosad & 9,6 & 20 & $4,2 \pm 1,07 \mathrm{abc}$ & 48,8 \\
\hline Tracer 480 SC & Spinosad & 12,0 & 25 & $2,0 \pm 0,77 \mathrm{abcd}$ & 75,6 \\
\hline Intrepid 240 SC & Metoxifenozide & 9,6 & 40 & $0,6 \pm 0,60 \mathrm{~d}$ & 92,7 \\
\hline Intrepid 240 SC & Metoxifenozide & 14,4 & 60 & $0,2 \pm 0,20 \mathrm{~d}$ & 97,6 \\
\hline Proclaim 5 SG & Benzoato de emamectina & 0,05 & 10 & $1,2 \pm 0,37 \mathrm{~d}$ & 85,4 \\
\hline Proclaim 5 SG & Benzoato de emamectina & 0,075 & 15 & $0,8 \pm 0,37 \mathrm{~d}$ & 90,2 \\
\hline Lorsban 480 SC & Clorpirifós-etil & 72,0 & 150 & $0,2 \pm 0,20 \mathrm{~d}$ & 97,6 \\
\hline Testemunha & - & - & - & $8,2 \pm 2,48 \mathrm{a}$ & - \\
\hline
\end{tabular}

${ }^{1}$ Avaliação de 100 frutos por repetição, com cinco repetições por tratamento.

${ }^{2}$ Médias não seguidas pela mesma letra diferem entre si pelo teste de Tukey, em nível de 5\% de probabilidade de erro.

\section{REFERÊNCIAS}

ABBOTT, W.S. A method of computing the effectiveness of an insecticide. Journal of Economic Entomologic, v.18, p.265-267, 1925.

ARIOLI, C.J.; BOTTON, M.; CARVALHO, A.C. Controle químico da Grapholita molesta (Busck) (Lepidoptera: Tortricidae) na cultura do pessegueiro. Ciência Rural, v.34, p.1695-1700, 2004.

BOTTON, M.; NAKANO, O.; KOVALESKI, A. Controle químico da lagarta-enroladeira (Bonagota cranaodes, Meyrick) na cultura da macieira. Pesquisa Agropecuária Brasileira. v.35, p.139-2144, 2000.

BROWN, J.W.; RAZOWSKI, J. Description of Ptychocroca, a new genus from Chile and Argentina, with comments on the Bonagota Razowski group of genera (Lepidoptera: Tortricidae: Euliini). Zootaxa, v.303, p.131, 2003.

CARLSON, G.R.; HADIALLA, T.S.; HUNTER, R.; JANSSON, R.K.; JANY, C.S.; LIBERT, Z.; SLAWECKI, R.A. The chemical and biological properties of methoxyfenozide, a new insecticidal ecdysteroid agonist. Pest Management Science, v.57, p.115-119, 2001.

CRUZ, C.D. Programa Genes: Aplicativo computacional em genética e estatística. Viçosa: Editora UFV, 2001. 648 p.

HADIALLA, T.S.; CARLSON, G.R.; LE, D. New insecticides with ecdysteroidal and juvenile hormone activity. Annual Review of Entomology, v.43, p.545-569, 1998. JANSSON, R.K.; PETERSON, R.F.; MOOKERJEE, P.K.; HALLIDAY, W.R.; ARGENTINE, J.A.; DYBAS, R.A.
Efficacy of solid formulation of emamectin benzoate controlling lepidopterous pest. Florida Entomologist, v.79, p. 434-449, 1997.

KOVALESKI, A. Eficiência de inseticidas no controle da lagarta-enroladeira (Phtheocroa cranaodes) em condições de laboratório. Horti Sul, v.3, p.30-32, 1994.

KOVALESKI, A.; BOTTON, M.; EIRAS, A.E.; VILELA, E. Lagarta-enroladeira da macieira: bioecologia e controle. Bento Gonçalves: Embrapa-Uva e Vinho, 1998. 22p. (Circular Técnica, 24).

KOVALESKI, A.; RIBEIRO, L.G. Manejo de pragas na produção integrada de maçã. Bento Gonçalves: EMBRAPA-CNPUV, 2002. 8p.

KOVALESKI, A.; RIBEIRO, L.G. Manejo de pragas na produção integrada de maçã. In: PROTAS, J.F. da S.; SANHUEZA, R.M.V. (Org.). Produção integrada de frutas: o caso da maçã no Brasil. Bento Gonçalves: Embrapa Uva e Vinho, 2003. 192p.

LEIBEE, G.L.; JANSSON, R.K.; NUESSLY, G.; TAYLOR, J.L. Efficacy of emamectin benzoate and Bacillus thuringiensis at controlling diamondback moth (Lepidoptera: Plutellidae) populations on cabbage in Florida. Florida Entomologist, v.78, p.82-96, 1995.

LORENZATO, D. Ensaio laboratorial de controle da "traça-da-maçã" Phtheochroa cranaodes Meyrick, 1937 com Bacillus thuringiensis Berliner e inseticidas químicos. Agronomia Sul-Rio-Grandense, v.20, p.157-163, 1984. MORANDO, A.; BEVIONE, D.; MORINO, G. Prove di controlle delle tignole della vite côn prodotti tradizionali e regolatori di crescita. L'Informatore Agrario, v.16, p.141-145, 1990. 
PARRA, J.R.P.; EIRAS, A.E.; HADDAD, M.L.; VILELA, E.F.; KOVALESKI, A. Técnica de criação de Phtheocroa cranaodes Meyrick (Lepidoptera: Tortricidae) em dieta artificial. Revista Brasileira de Biologia, v.55, n.4, p.537543, 1995.

PASTORI, P.L. Bioecologia de Trichogramma pretiosum (Riley, 1879) (Hymenoptera: Trichogrammatidae) e Controle Integrado de Bonagota salubricola (Meyrick, 1937) e Grapholita molesta (Busck, 1916) (Lepidoptera: Tortricidae) com feromônios sexuais na cultura da macieira. 2007. 151f. Dissertação (Mestrado em Ciências Biológicas-Entomologia) - Universidade Federal do Paraná, Curitiba, 2007.

PROTAS, J. F. S.; SANHUEZA, R.M.V. Normas técnicas e documentos de acompanhamento da produção integrada de maçã. Bento Gonçalves, Embrapa Uva e Vinho, 2000. $64 \mathrm{p}$.

SCOZ, P.L.; BOTTON, M.; GARCIA, M.S. Controle químico de Anastrepha fraterculus (Wied.) (Diptera: Tephritidae) em laboratório. Ciência Rural, v.34, p.1689$1694,2004$.

SPARKS, T.C.; THOMPSON, G.D.; KIRST, H.A.; HERTLEIN, M.B.; LARSON, L.L.; WORDEN, T.V.;
THIBAULT, S.T. Biological activity of the spinosys, new fermentation derived insect control agents, on tobacco budworm (Lepidoptera: Noctuidae) larvae. Journal Economic of Entomology, v.91, p.1277-1283, 1998.

SUN, X.; BARRETT, B.A.; BIDDINGER, D.J. Fecundidad and fertility changes in adult leafrollers exposed to surfaces treated with ecdysteroid agonists tebufenozide and methoxyfenozide. Entomologia Experimentalis et Applicata, v.94, p.75-83, 2000.

THOMPSON, G.; HUTCHINS, S. Spinosad. Pesticide Outlook, v.10, p.78-81, 1999.

TITI, A.E.; BOLLER, E.F.; GENDRIER, J.P. Producción integrada: principios y directrices técnicas. [S.1.]: IOBC/ WPRS, 1995. 22p.

YOSHIMOTO, T.; OGAWA, S.; UDAGAWA, T.; NUMATA, S. Development of new inseticide, Etofenprox. Journal of Pesticide Science, v.14, p.259-268, 1989.

Recebido em 3/8/07

Aceito em 9/2/09 